\title{
Synthesis of controllable monodisperse gold nanoparticles using wood material and their catalytic activity for $p$-nitrophenol reduction
}

\author{
Xiaobo $\operatorname{Lin}^{1}$, Min $\mathrm{Wu}^{2}$, Shigenori Kuga ${ }^{2}$, Takashi Endo ${ }^{3}$ and Yong Huang ${ }^{2}$ \\ Monodisperse gold nanoparticles (Au NPs) with sizes between 2.3 and $23.1 \mathrm{~nm}$ were synthesized with the assistance of \\ a natural wood material as a reductant/dispersant. The average diameter of the Au NPs could be controlled by varying the \\ concentration of the Au precursor, and the size distribution could be improved by the addition of $\mathrm{NaOH}$. The different growing \\ processes of Au NPs with and without $\mathrm{NaOH}$ were comprehensively studied. The obtained wood-supported Au NPs showed high \\ catalytic activity for the reduction of $p$-nitrophenol as a model reaction. Moreover, Au NPs with smaller sizes exhibited higher \\ catalytic activity and the catalyst could be easily recovered via centrifugation for reuse.
}

Polymer Journal (2016) 48, 919-923; doi:10.1038/pj.2016.51; published online 25 May 2016

\section{INTRODUCTION}

Monodisperse gold nanoparticles (Au NPs) with tunable morphology are especially useful as novel materials for the detection of metal ions, glucose oxidation and X-ray attenuation and so on. ${ }^{1-3}$ Controlling the size and shape of monodisperse Au NPs is crucial due to the structure sensitivity in many fields, such as biochemistry, surface plasmon and catalysts. $^{2,4,5}$ Great efforts have been devoted to synthesizing Au NPs with uniform shape and size; examples of processes include the improved citrate reduction process, the polyol process and physical radiolytic synthesis. ${ }^{6-9}$ Moreover, hydroquinone, shortened singlewalled carbon nanotubes, diphosphine and organothiolate ligands and so on have been introduced as reducing or dispersing agents to prepare monodisperse Au NPs. ${ }^{4,10-12}$ However, some of the abovementioned methods use toxic-reducing agents and organic solvents, making them environmentally unfavorable. Therefore, there remains a need to establish eco-friendly routes for the preparation of Au NPs with controlled size and shape.

Biomass-based chemical processes have drawn much attention due to growing concern regarding the depletion of fossil fuels and emission of greenhouse gases. Many natural extracts, such as tannin, honey, folic acid and leaf broth, have been used in the biomass-based synthesis of Au NPs. ${ }^{13-16}$ However, the reported schemes are not satisfactory. First, most protocols require extraction or chemical modification of biomass. ${ }^{17}$ Second, the size and shape of Au NPs have not been controlled well. ${ }^{14}$ Thus, it has been difficult to synthesize monodisperse Au NPs via an eco-friendly and facile route.

It has been well documented that cellulose can be employed to fabricate metal NPs. Compared with cellulose, natural wood is a mixture that contains lignin and hemicellulose in addition to cellulose. The phenolic hydroxyl groups in lignin have a higher reducing ability than the hydroxyl groups in cellulose. Moreover, the secondary alcohol and ethers of hemicellulose also have the reducing and stabilizing ability for metal NPs. ${ }^{18-21}$ So far, there have been few reports on the precise control of the shape and size of Au NPs using wood materials. One of the main obstacles to using the chemical groups in wood is their poor accessibility. Thus, there is a need to increase the surface of the wood so that the functional groups in wood can become accessible.

In this paper, a wood material with a large surface area was obtained through mechanical grinding with water so that the functional groups in the wood could be utilized effectively. Both the solid and soluble wood components were effective for the controllable preparation of $\mathrm{Au}$ NPs. Without any extraction or chemical modification, the material could be used to synthesize monodisperse Au NPs. The obtained Au NPs had narrow size distribution compared with reported Au NPs synthesized using other biomass chemicals. Finally, the Au NP product was tested for the catalytic reduction of p-nitrophenol as a model reaction.

\section{EXPERIMENTAL PROCEDURE}

Synthesis

Japanese Hinoki wood was pulverized to pass through a 3-mm-sized sieve by a cutter mill (MKCM-3, Masuko Sangyo, Kawaguchi, Japan). The wood flour was dispersed in water to make a $5 \mathrm{wt} \%$ solid suspension. The suspension was ground by a disk mill (MKZA6-2, Masuko Sangyo) through 15-cycle treatments to produce the nano-wood material. The water suspension was treated with a

\footnotetext{
${ }^{1}$ Shijiazhuang Tiedao University, Hebei Provincial Key Laboratory of Traffic Engineering materials, Hebei, China; ${ }^{2}$ Technical Institute of Physics and Chemistry, Chinese Academy of Sciences, Beijing, China and ${ }^{3}$ Research Institute for Sustainable Chemistry, National Institute of Advanced Industrial Science and Technology, Hiroshima, Japan Correspondence: Professor M Wu or Dr Y Huang, Technical Institute of Physics and Chemistry, Chinese Academy of Sciences, 29 Zhongguancun East Road, Beijing 100190, China.

E-mail: wumin@mail.ipc.ac.cn or yhuang@mail.ipc.ac.cn

Received 9 December 2015; revised 25 February 2016; accepted 22 March 2016; published online 25 May 2016
} 
tip sonicator (Ultrasound Cell Crusher, Scientz-08, Ningbo, Jiangsu province, China) before use. Auric chloride acid $\left(\mathrm{HAuCl}_{4}\right.$, Shenyang Jinke Reagent, Liaoning province, China) was used as received.

In a typical procedure, a certain volume of $0.01 \mathrm{M} \mathrm{HAuCl}_{4}$ solution was added to $10 \mathrm{ml}$ of $0.1 \mathrm{wt} \%$ nano-wood material suspension, and the mixture was stirred at various temperatures for $1 \mathrm{~h}$. The reaction was also carried out with the addition of various volumes of $1.0 \mathrm{M} \mathrm{NaOH}$ solution.

\section{Surface area analysis by nitrogen adsorption}

The nano-wood materials were subjected to solvent-exchange drying with $n$-butyl alcohol and then freeze-dried. Nitrogen adsorption measurements of the dried samples were performed using a Quantachrome NOVA 4000 (Yuasa Ionics, Tokyo, Japan). Specific surface areas were obtained via capillary condensation analysis using the accompanying software.

\section{UV-visible absorption of Au NPs}

An aliquot of the reaction mixture was taken out and UV-visible absorption spectroscopy of the Au NPs was recorded using a spectrophotometer (Cary 5000).

\section{Transmission electron microscopy}

The morphology of Au NPs was observed using a Hitachi H-800 transmission electron microscope (TEM) operated at $100 \mathrm{keV}$ and a JEOL FS-2200 highresolution transmission electron microscope (HRTEM) operated at $200 \mathrm{keV}$. The specimens for TEM and HRTEM observations were prepared by depositing a drop of suspension onto carbon-coated copper grids and drying in air. The number-averaged diameter of Au NPs was measured for more than 100 particles using the TEM and HRTEM images.

\section{Catalytic activity}

After performing catalytic reactions, the Au NP-containing nano-wood particles were sedimented via centrifugation, and the supernatant was analyzed via inductively coupled plasma atomic emission spectrometry (Varian $710 \mathrm{ES}$ ). The loading of the Au NPs was calculated using the initial amount of the Au precursor and its remaining amount after reductive deposition. One milliliter of aqueous solution of $p$-nitrophenol $(1.3 \mathrm{~mm})$ and sodium borohydride $\left(\mathrm{NaBH}_{4}\right.$, $0.42 \mathrm{M}$ ) was prepared and mixed with $6 \mathrm{ml}$ of water. The addition of $1 \mathrm{ml}$ of $\mathrm{NaBH}_{4}$ solution to it caused an immediate yellow coloration. Then, $2 \mathrm{ml}$ of the $\mathrm{Au} \mathrm{NP}$ catalyst suspension was injected into the system $\left(4.5 \times 10^{-4} \mathrm{M}\right)$ and $1 \mathrm{ml}$ of the mixed solution was quickly filtered through a $0.22 \mu \mathrm{m}$ filter before measurements. The filtrate $(0.8 \mathrm{ml})$ was added to $2 \mathrm{ml}$ of water and put into a quartz cuvette and the absorption at $400 \mathrm{~nm}$ was recorded.

\section{RESULTS AND DISCUSSION}

After mechanical treatment with water, a yellow wood suspension was obtained. This suspension functions as both a reducing and dispersing agent for $\mathrm{Au}$ ions and $\mathrm{Au}$ NPs. The reduction of $\mathrm{Au}$ ions by wood is a complex process. It has been reported that primary alcohols, secondary alcohols and diethers are available as reductants for the preparation of metal colloidal dispersions. All of the phenol hydroxyl groups of lignin, the hydroxyl of cellulose and secondary alcohol and ethers of hemicellulose have reducing ability for the preparation of $\mathrm{Au}$ NPs. Meanwhile, the dispersant effect of wood suspensions for Au NPs works two ways. The solid part of the wood suspension has a nanofibrillar structure, as observed by TEM (Figure 1). The large specific surface areas of the solid lignocelluloses fibers $\left(143 \mathrm{~m}^{2} \mathrm{~g}^{-1}\right.$, as determined by BET) make them a suitable loading medium for the deposition of Au NPs. Here, the hydroxyl groups on the surface of the lignocelluloses serve as the depositing substrate. At the same time, some water-soluble components derived from hemicelluloses in wood may act as the dispersion solution. ${ }^{22}$

The reduction of $\mathrm{Au}$ ions by nano-wood materials at room temperature requires a long time, approximately $8 \mathrm{~h}$ or more. When

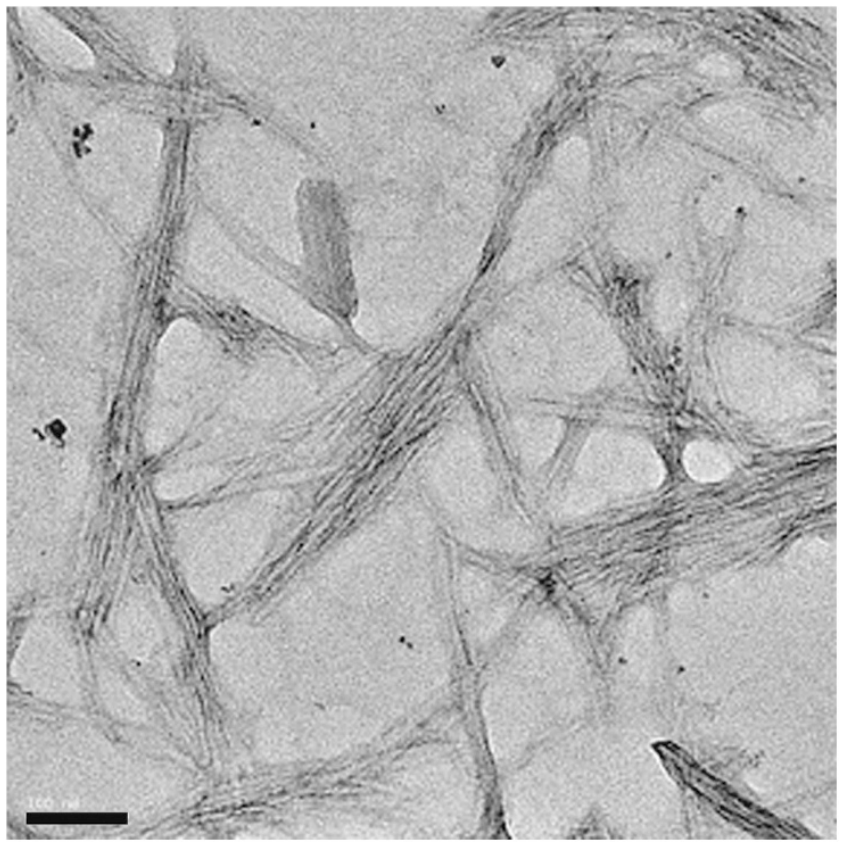

Figure 1 Transmission electron microscope image of wood nanofiber obtained by grinding. Scale bar, $100 \mathrm{~nm}$.
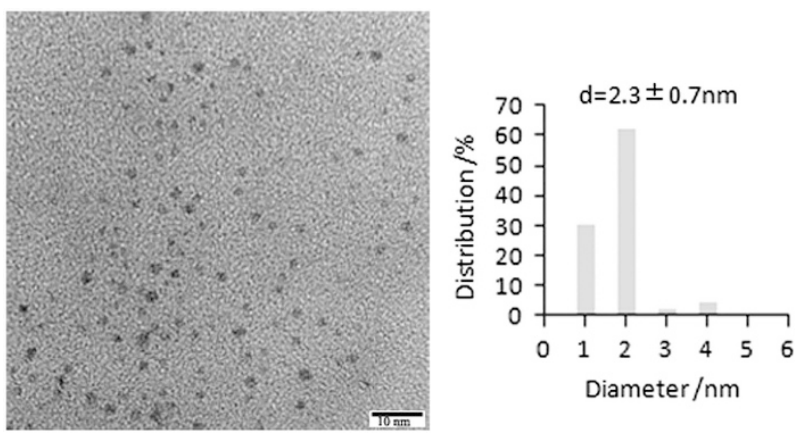

Figure 2 Transmission electron microscope images of monodisperse Au nanoparticles reduced by nano-wood material and their size histogram. Starting concentration of $\mathrm{HAuCl}_{4}$ is $0.03 \mathrm{~mm}$. Scale bar, $10 \mathrm{~nm}$.

heated to $100{ }^{\circ} \mathrm{C}$, the reaction time shortens significantly. The following results refer to a reaction temperature of $100^{\circ} \mathrm{C}$ unless stated otherwise. Higher temperature treatments, however, tended to result in aggregation of the particles. The solid content of the suspension was $0.1 \mathrm{wt} \%$, and an $\mathrm{Au}^{3+}$ concentration of $0.03 \mathrm{~mm}$ was examined. The reaction under these conditions produced monodisperse spherical Au NPs with a diameter of $2.3 \pm 0.7 \mathrm{~nm}$ (Figure 2). When the concentration of $\mathrm{Au}^{3+}$ increased, the particles converted to sheet shapes (Figure 3a). This concentration dependence of particle size and shape is probably due to the step-by-step crystal growth, which has been generally observed in metal NP formation. ${ }^{23}$ In our protocol, some water-soluble components derived from hemicelluloses in wood may act as the stabilizer, which could control the formation of the different morphologies of Au NPs. ${ }^{22}$

It has been reported that homogeneous reduction leads to an $\mathrm{Au}$ particle whose shape is affected by the solution conditions, such as the $\mathrm{pH}$, type of reductant and precursor concentration. ${ }^{24-26}$ The reducing 

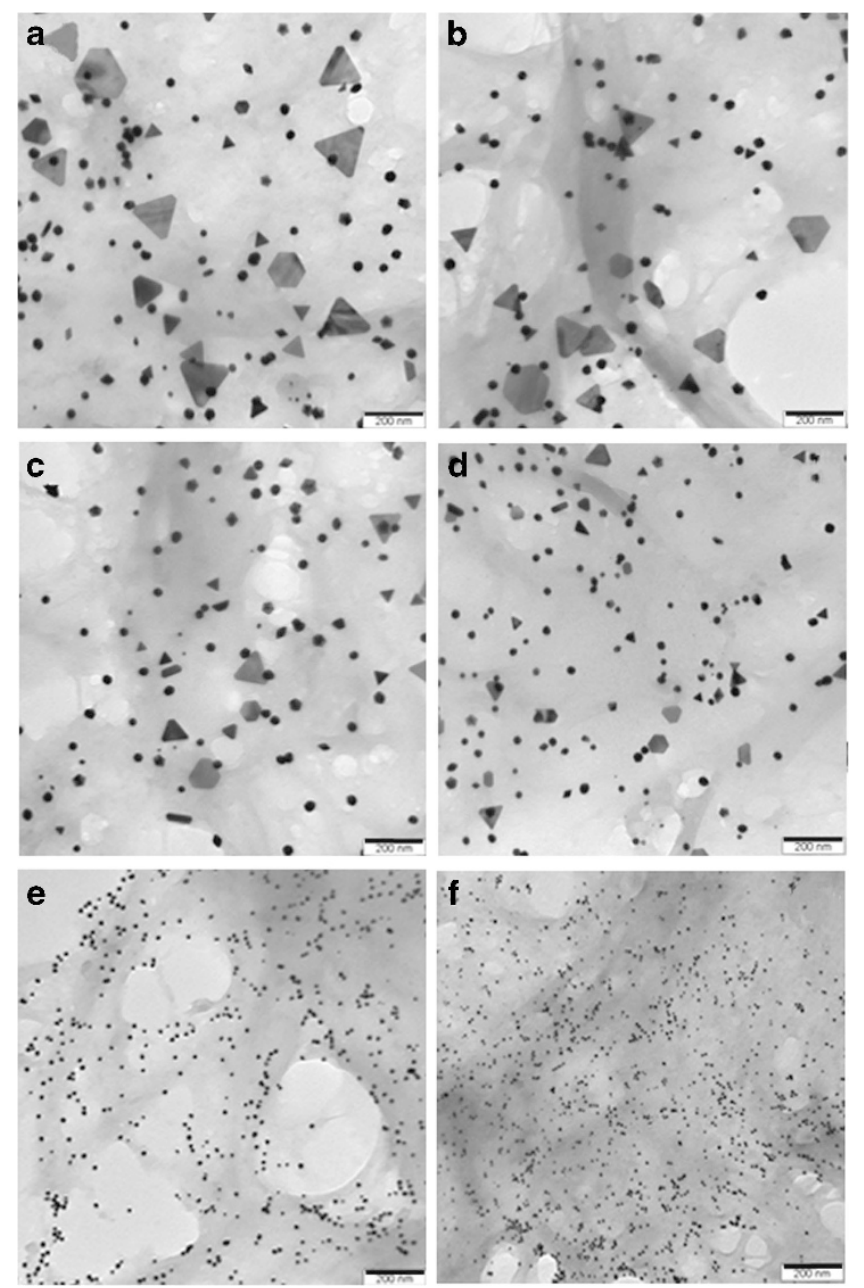

Figure 3 Transmission electron microscope images of $\mathrm{Au}$ nanoparticles reduced by nano-wood materials with a $\mathrm{HAuCl}_{4}$ concentration of $0.3 \mathrm{~mm}$ and different mole ratios of $\mathrm{OH}^{-} / \mathrm{Au}^{3+}$ : (a) $0: 1$, (b) $1: 1$, (c) $2: 1$, (d) $3: 1$, (e) $4: 1$, (f) $5: 1$. Scale bar, $200 \mathrm{~nm}$.

agent in our experiment, that is, nano-wood, is a heterogeneous suspension. The influence of the $\mathrm{pH}$ on the heterogeneous preparation of $\mathrm{Au}$ NPs was studied in detail. By varying the mole ratio of $\mathrm{OH}^{-} / \mathrm{Au}^{3+}$ under set concentrations of $\mathrm{Au}^{3+}$, remarkable differences in particle size and shape were observed. The TEM images (Figure 3a) show that without the addition of $\mathrm{OH}^{-}$, triangular and hexagonal $\mathrm{Au}$ nanoplates were formed. The UV-visible absorption spectrum of the sample shows two strong absorption peaks centered at 530 and $583 \mathrm{~nm}$ (Figure 4). According to the Mie theory, small spherical Au nanocrystals will show only a single absorption peak, whereas anisotropic particles can show two or three absorption peaks depending on their shape. The spherical Au NPs in the suspension might contribute to the absorption peak at approximately $530 \mathrm{~nm}$, but the in-plane dipole absorption peak is a unique feature of the Au nanoplates. With increased addition of $\mathrm{OH}^{-}$, the shoulder peak at approximately $583 \mathrm{~nm}$ decreased, while the $530 \mathrm{~nm}$ peak increased (Figure 4). The TEM images showed that the number of nanoplates decreased greatly as $\mathrm{OH}^{-}$increased. The nanoplates disappeared with a mole ratio of $\mathrm{OH}^{-} / \mathrm{Au}^{3+}$ of $4: 1$ and 5:1. At the same time, the sizes of spherical particles decreased and became nearly homogeneous (Figures $3 \mathrm{e}-\mathrm{f}$ ). The average size was 11.5 and $9.8 \mathrm{~nm}$.

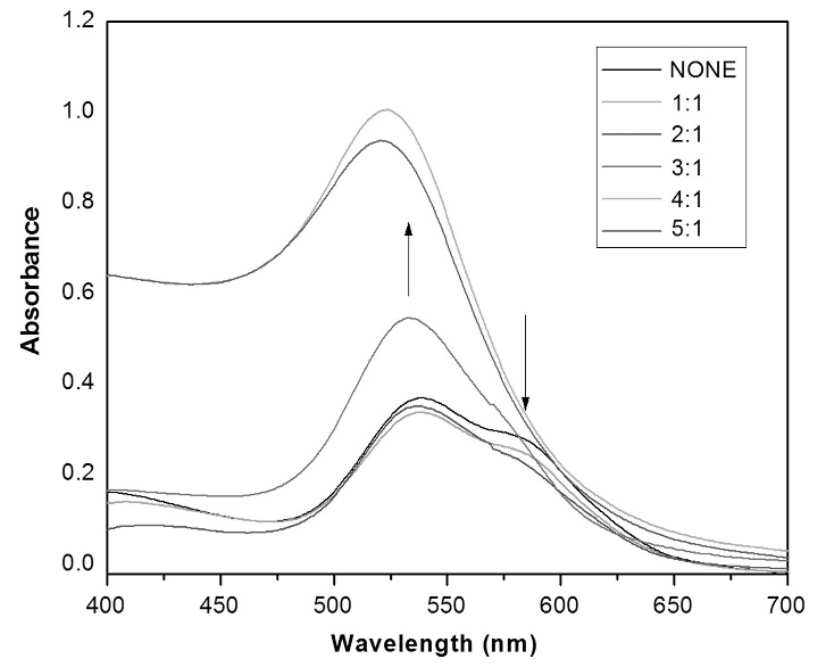

Figure 4 UV-visible (UV-vis) absorption spectra of Au nanoparticles reduced by nano-wood material. $\mathrm{HAuCl}_{4}$ of $0.3 \mathrm{~mm}$ at different mole ratios of $\mathrm{OH}^{-} / \mathrm{Au}^{3+}$, without the addition of $\mathrm{NaOH}$, from $1: 1$ to $5: 1$. Vertical arrows indicate that the UV-vis absorption peak decreased by approximately $583 \mathrm{~nm}$ and increased by approximately $530 \mathrm{~nm}$ with a higher mole ratio of $\mathrm{OH}^{-} / \mathrm{Au}^{3+}$. A full color version of this figure is available at Polymer Journal online.

The strong influences of alkali addition on the growth and size distribution of $\mathrm{Au}$ NPs can be utilized to prepare monodisperse $\mathrm{Au}$ NPs of desired sizes. As shown in Figure 5, the $\mathrm{Au}$ precursor concentration was increased from 0.3 to $3.0 \mathrm{~mm}$ under an $\mathrm{OH}^{-} / \mathrm{Au}^{3+}$ ratio of $5: 1$, with the average diameter of Au NPs changing from $11.1 \pm 1.1 \mathrm{~nm}$ to $23.1 \pm 2.2 \mathrm{~nm}$. The UV-visible absorption spectra showed corresponding changes (Figure 6), that is, the absorption peak shifted from 521 to $547 \mathrm{~nm}$.

The temporal evolution of Au NPs reduced by the nano-wood suspension was studied to clarify the mechanism of the growth of $\mathrm{Au}$ nanocrystals under different $\mathrm{OH}^{-} / \mathrm{Au}^{3+}$ ratios. Supplementary Figure s1 shows the temporal size evolution of Au NPs when the $\mathrm{Au}^{3+}$ concentration was $0.3 \mathrm{~mm}$ without the addition of $\mathrm{OH}^{-}$. The TEM image indicates that the particles obtained after a reaction time of $10 \mathrm{~s}$ were still small in size. After $1 \mathrm{~min}$, the Au NPs were overlapping, and some wire-like aggregates could be observed (Supplementary Figure s1). After $2 \mathrm{~min}$, the wire-like structure disappeared, and the particle sizes increased dramatically, indicating a rapid autocatalytic process catalyzed by the nuclei and crystal growth of $\mathrm{Au}$ NPs. After reacting for $10 \mathrm{~min}$, triangular Au nanoplates were formed; the same morphology could be observed after $60 \mathrm{~min}$, indicating the ripening of the $\mathrm{Au}$ nanocrystal. Supplementary Figure s2 shows the temporal size evolution of Au NPs when the concentration of $\mathrm{Au}^{3+}$ was $0.3 \mathrm{~mm}$ and the mole ratio of $\mathrm{OH}^{-}$to $\mathrm{Au}^{3+}$ was 5:1. After the first $30 \mathrm{~s}$, small, spherical particles were formed. After $10 \mathrm{~min}$, the particles were still spherical in shape, with a narrow size distribution. This result illustrates that in this heterogeneous reduction process, the addition of $\mathrm{NaOH}$ could greatly affect the reaction mechanism, which is crucial to the morphology and stabilization of monodisperse Au NPs. It is well documented that the stabilizing agents have a crucial role in the shape-controlled syntheses of metal NPs under different pHs. ${ }^{22,24,27,28}$ The stabilizing agent could adsorb onto the Au NP surface and provide steric barriers for repulsion interactions, which could reduce the aggregation of particles. In the low $\mathrm{pH}$ range, as shown in Supplementary Figure s1, some overlapping, wire-like aggregate and Au nanoplates are the main 

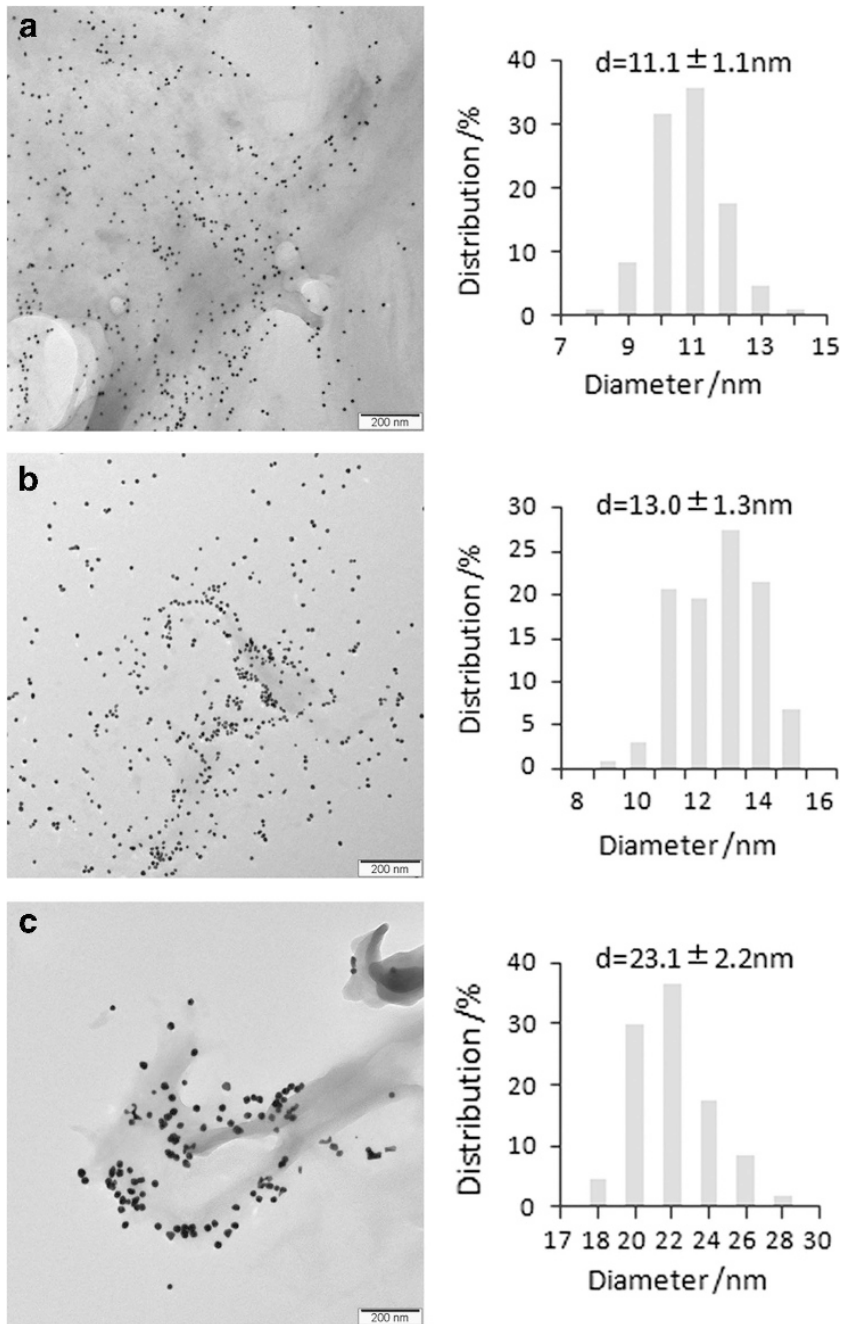

Figure 5 (a-c) Left: transmission electron microscope images of $\mathrm{Au}$ nanoparticles reduced by nano-wood material, with $\mathrm{OH}^{-} / \mathrm{Au}^{3+}$ of $5: 1$, at different $\mathrm{HAuCl}_{4}$ concentrations; (a) $0.3 \mathrm{~mm}$, (b) $0.6 \mathrm{~mm}$, (c) $3.0 \mathrm{~mm}$. Right: particle size distribution. Scale bar, $200 \mathrm{~nm}$.

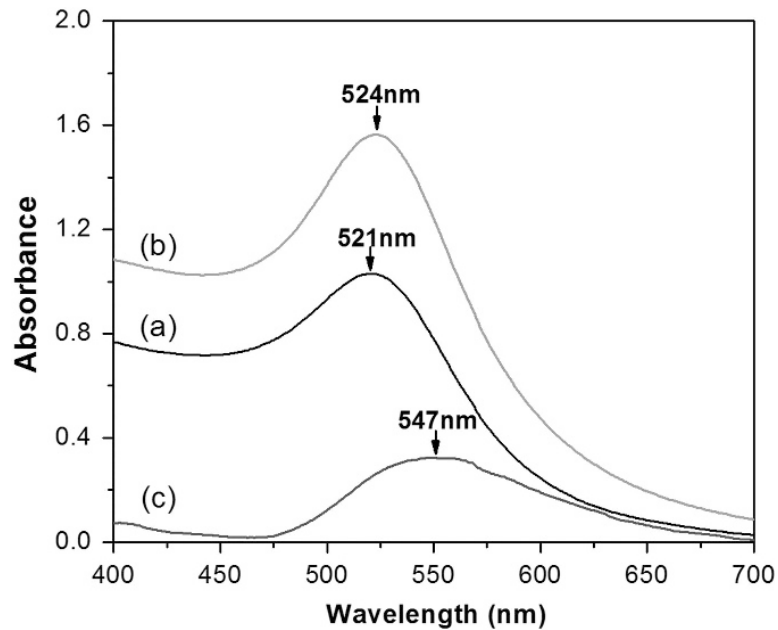

Figure 6 UV-visible absorption spectra of Au nanoparticles reduced by nanowood material, with $\mathrm{OH}^{-} / \mathrm{Au}^{3+}$ of $5: 1$, at different $\mathrm{HAuCl}_{4}$ concentrations; (a) $0.3 \mathrm{~mm}$, (b) $0.6 \mathrm{~mm}, \quad$ (c) $3.0 \mathrm{~mm}$. A full color version of this figure is available at Polymer Journal online.
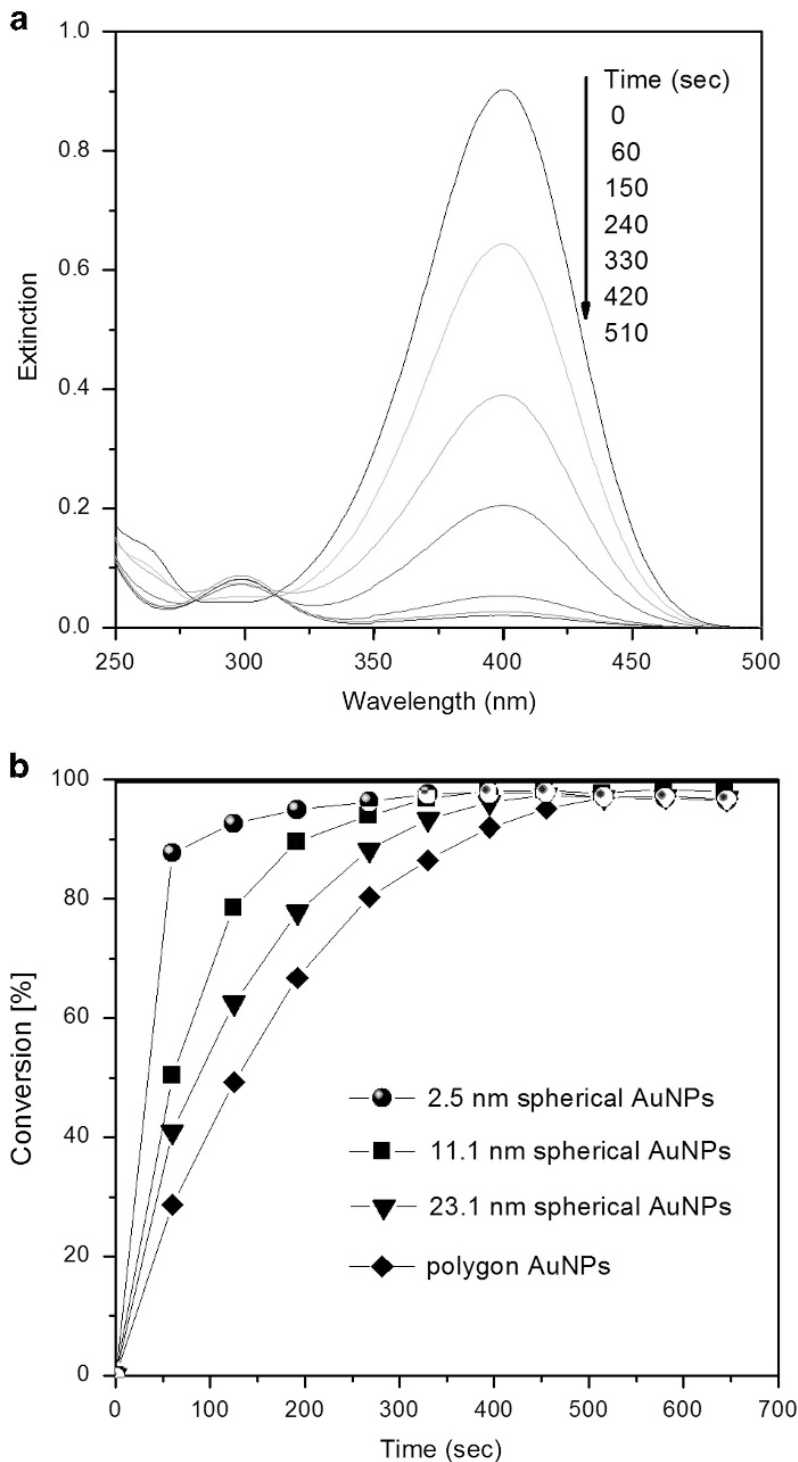

Figure 7 (a) Time course change in UV-visible spectra of p-nitrophenol solution catalyzed by Au nanoparticles (NPs) in Figure 3a. (b) Intensity time course of $400 \mathrm{~nm}$ peak for product ( $p$-nitrophenol). A full color version of this figure is available at Polymer Journal online.

structures of Au particles. The tendency of particles to form aggregates may be the lack of effective stabilizing agent. When more $\mathrm{OH}^{-}$was added, the particles remained a distance away from each other during the nucleation and growth process (Supplementary Figure s2). In our protocol, some water-soluble components derived from hemicelluloses in wood may act as the capping agent for Au NPs. ${ }^{22}$ The hydrolysis of the hemicelluloses under higher $\mathrm{OH}^{-} / \mathrm{Au}^{3+}$ ratios may affect the shape evolution of Au NPs. Although further work is required to clarify this phenomenon, the $\mathrm{pH}$-sensitive structures of the hydrolysis products of the hemicellulose may be one of the key reasons for why the aggregation stops.

The catalytic activity of nano-wood-supported Au NPs was tested for the reduction of $p$-nitrophenol by $\mathrm{NaBH}_{4}$, which is a standard model reaction for estimating the catalytic activity of Au NPs. First, it was confirmed that the wood nanomaterial had no adsorption capacities for $p$-nitrophenol and that the reduction does not proceed without catalyst. The addition of the nano-wood- 
supported Au NPs to the reaction mixture caused rapid fading of the yellow color. This color change was used to monitor the reaction kinetics based on spectroscopic measurements. Figure 7 shows a typical change in the UV-visible spectra caused by the reduction of $p$-nitrophenol by the supported Au NPs (the sample shown in Figure 3a, obtained from $0.3 \mathrm{~mm} \mathrm{HAuCl}_{4}$ ). The absorption by $p$-nitrophenol at $400 \mathrm{~nm}$ decreased with concomitant appearance of the $290 \mathrm{~nm}$ peak of $p$-aminophenol within $10 \mathrm{~min}$. For all the experiments, the initial concentrations of $p$-nitrophenol and $\mathrm{NaBH}_{4}$ were kept at $1.3 \times 10^{-4}$ and $4.2 \times 10^{-2} \mathrm{M}$, respectively. The concentrations of the Au-based catalyst were $4.5 \times 10^{-4} \mathrm{M}$ to keep the number of Au atoms at the same level. As shown in Figure $7 \mathrm{~b}$, the catalytic activity was in the following order: spherical $\mathrm{Au} \quad \mathrm{NPs} \quad(2.3 \pm 0.7 \mathrm{~nm})>\mathrm{Au} \quad \mathrm{NPs} \quad(11.1 \pm 1.1 \mathrm{~nm})>\mathrm{Au} \quad \mathrm{NPs}$ $(23.1 \pm 2.1 \mathrm{~nm})>$ polygon Au NPs (the sample shown in Figure $3 \mathrm{a}$ ). Thus, the catalytic activity was negatively dependent on the particle size. This result agrees with the reports that Au NPs with larger accessibility surfaces are catalytically more active. ${ }^{28,29}$ Our protocol demonstrated that wood nanomaterials, a type of natural biomass mixture, could be used as eco-friendly supports for an $\mathrm{Au}$ catalyst in the reduction of $p$-nitrophenol. The catalyst could be easily recovered via centrifugation for reuse, nearly retaining its activity. Only a slight decrease in activity was observed in the third cycle (Supplementary Table S1). A possible cause of this deterioration is the release of $\mathrm{Au}$ particles from the nano-wood support. Further work is required to clarify this degradation of catalytic activities in the present system.

\section{CONCLUSIONS}

We demonstrated here that nanomaterial derived from natural wood is useful as a reducing agent and support for monodisperse Au NPs. The concentrations of $\mathrm{Au}$ precursor and hydroxyl ions are the governing factors in the controlled synthesis of monodisperse $\mathrm{Au}$ NPs. The obtained Au NPs supported by nano-wood material showed high catalytic activity in the reduction of $p$-nitrophenol. The procedure developed here offers a potentially useful route for utilizing plantbased functional nanomaterials.

\section{CONFLICT OF INTEREST}

The authors declare no conflict of interest.

\section{ACKNOWLEDGEMENTS}

This work was supported by the National Program on Key Basic Research Project (973Program, No. 2011CB933700), the National Natural Science Foundation of China (51172247, 51043003, 50773086, 51102172), the Chinese Academy of Sciences Visiting Professorships and Hebei Key Discipline Construction Project.

1 Mao, X., Li, Z. \& Tang, Z. One pot synthesis of monodispersed L-glutathione stabilized gold nanoparticles for the detection of $\mathrm{Pb} 2+$ ions. Front. Mater. Sci. 5, 322-328 (2011).

2 Wang, J. F., Gong, J. X., Xiong, Y. S., Yang, J. D., Gao, Y., Liu, Y. L., Lu, X. Q. \& Tang, Z. Y. Shape-dependent electrocatalytic activity of monodispersed gold nanocrystals toward glucose oxidation. Chem. Commun. (Camb) 47, 6894-6896 (2011).

3 Wang, Z. J., Wu, L. N. \& Cai, W. Size-tunable synthesis of monodisperse water-soluble gold nanoparticles with high X-ray attenuation. Chemistry 16, 1459-1463 (2010).
4 Parker, J. F., Fields-Zinna, C. A. \& Murray, R. W. The story of a monodisperse gold nanoparticle: Au25L18. Acc. Chem. Res. 43, 1289-1296 (2010).

5 Tang, R., Liao, X. P. \& Shi, B. Heterogeneous gold nanoparticles stabilized by collagen and their application in catalytic reduction of 4-nitrophenol. Chem. Lett. 37, 834-835 (2008).

6 Pimpang, P. \& Choopun, S. Monodispersity and stability of gold nanoparticles stabilized by using polyvinyl alcohol. Chiang Mai J. Sci. 38, 31-38 (2011).

7 Hiramatsu, H. \& Osterloh, F. E. A simple large-scale synthesis of nearly monodisperse gold and silver nanoparticles with adjustable sizes and with exchangeable surfactants. Chem. Mater. 16, 2509-2511 (2004).

8 Jana, N. R. \& Peng, X. Single-phase and gram-scale routes toward nearly monodisperse $\mathrm{Au}$ and other noble metal nanocrystals. J. Am. Chem. Soc. 125 , 14280-14281 (2003).

9 Henglein, A. \& Meisel, D. Radiolytic control of the size of colloidal gold nanoparticles. Langmuir 14, 7392-7396 (1998).

10 Perrault, S. D. \& Chan, W. C. W. Synthesis and surface modification of highly monodispersed, spherical gold nanoparticles of 50-200 nm. J. Am. Chem. Soc. 131, 17042-17043 (2009).

11 Pettibone, J. M. \& Hudgens, J. W. Synthetic approach for tunable, size-selective formation of monodisperse, diphosphine-protected gold nanoclusters. J. Phys. Chem. Lett. 1, 2536-2540 (2010).

12 Kim, J.-W., Moon, H.-M., Benamara, M., Sakon, J., Salamo, G. J. \& Zharov, V. P. Aqueous-phase synthesis of monodisperse plasmonic gold nanocrystals using shortened single-walled carbon nanotubes. Chem. Commun. (Camb) 46, 7142-7144 (2010).

13 Huang, X., Wu, H., Liao, X. P. \& Shi, B. One-step, size-controlled synthesis of gold nanoparticles at room temperature using plant tannin. Green Chem. 12, 395-399 (2010).

14 Philip, D. Honey mediated green synthesis of gold nanoparticles. Spectrochim. Acta A Mol. Biomol. Spectrosc. 73, 650-653 (2009).

15 Li, G., Li, D., Zhang, L., Zhai, J. \& Wang, E. One-step synthesis of folic acid protected gold nanoparticles and their receptor-mediated intracellular uptake. Chemistry 15 9868-9873 (2009).

16 Smitha, S. L., Philip, D. \& Gopchandran, K. G. Green synthesis of gold nanoparticles using Cinnamomum zeylanicum leaf broth. Spectrochim. Acta A Mol. Biomol. Spectrosc. 74, 735-739 (2009).

17 Nadagouda, M. N. \& Varma, R. S. Green and controlled synthesis of gold and platinum nanomaterials using vitamin B-2: density-assisted self-assembly of nanospheres, wires and rods. Green Chem. 8, 516-518 (2006).

18 Talmadge, K. W., Keegstra, K., Bauer, W. D. \& Albershe, P. Structure of plant-cell walls.1. macromolecular components of walls of suspension-cultured sycamore cells with a detailed analysis of pectic polysaccharides. Plant Physiol. 51, 158-173 (1973).

19 Bauer, W. D., Talmadge, K. W., Keegstra, K. \& Albershe, P. Structure of plant-cell walls.2. hemicellulose of walls of suspension-cultured sycamore cells. Plant Physiol. 51, 174-187 (1973).

20 Lin, X. B., Wang, F. Q., Kuga, S., Endo, T., Wu, M., Wu, D. Y. \& Huang, Y. Eco-friendly synthesis and antibacterial activity of silver nanoparticles reduced by nano-wood materials. Cellulose 21, 2489-2496 (2014).

21 Lin, X., Wu, M., Wu, D., Kuga, S., Endo, T. \& Huang, Y. Platinum nanoparticles using wood nanomaterials: eco-friendly synthesis, shape control and catalytic activity for p-nitrophenol reduction. Green Chem. 13, 283-287 (2011).

22 Peng, H., Yang, A. S. \& Xiong, J. H. Green, microwave-assisted synthesis of silver nanoparticles using bamboo hemicelluloses and glucose in an aqueous medium. Carbohydr. Polym. 91, 348-355 (2013).

23 Nadagouda, M. N., Polshettiwar, V. \& Varma, R. S. Self-assembly of palladium nanoparticles: synthesis of nanobelts, nanoplates and nanotrees using vitamin B-1, and their application in carbon-carbon coupling reactions. J. Mater. Chem. 19, 2026-2031 (2009).

24 Shou, Q. H., Guo, C., Yang, L. R., Jia, L. W., Liu, C. Z. \& Liu, H. Z. Effect of pH on the single-step synthesis of gold nanoparticles using PEO-PPO-PEO triblock copolymers in aqueous media. J. Colloid Interface Sci. 363, 481-489 (2011).

$25 \mathrm{Ji}$, X. H., Song, X. N., Li, J., Bai, Y. B., Yang, W. S. \& Peng, X. G. Size control of gold nanocrystals in citrate reduction: the third role of citrate. J. Am. Chem. Soc. 129, 13939-13948 (2007).

26 Wang, Y., Ren, J. W., Deng, K., Gui, L. L. \& Tang, Y. Q. Preparation of tractable platinum, rhodium, and ruthenium nanoclusters with small particle size in organic media. Chem. Mater. 12, 1622-1627 (2000).

27 Chen, L., Xiao-Yue, Z. \& Chun-Lei, M. Rapid fabrication of gold nanoflowers tuned by $\mathrm{pH}$ : insights into the growth mechanism. J. Nanosci. Nanotechnol. 15 2761-2769 (2015).

28 Zeng, J., Zhang, Q., Chen, J. Y. \& Xia, Y. N. A comparison study of the catalytic properties of Au-based nanocages, nanoboxes, and nanoparticles. Nano Lett. 10, 30-35 (2010).

29 Panigrahi, S., Basu, S., Prahara, S., Pande, S., Jana, S., Anjali Pal, S. K., Ghosh \& Pal, T. Synthesis and size-selective catalysis by supported gold nanoparticles: study on heterogeneous and homogeneous catalytic process. J. Phys. Chem. C 111, 4596-4605 (2007). 